\title{
CRÓNICA DEL CONGRESO INTERNACIONAL E INTERDISCIPLINAR ITALO-ESPAÑOL 'L'UNIONE EUROPEA, IL DIRITTO PROCESSUALE E IL DIRITTO AMMINISTRATIVO LOCALE: LA DIFESA DEI DIRITTI E DEGLI INTERESSI DEI CITTADINI". BOLONIA (ITALIA), 25 Y 26 DE MAYO DE 2017
}

\author{
Pedro Manuel Quesada López \\ Contratado Predoctoral de Investigación FPU del MECD. Ref. FPU014/04912 \\ Área de Derecho Procesal. Universidad de Jaén \\ email:pquesada@ujaen.es
}

Rubén López Picó

Becario de Formación Interna de Derecho Procesal. Universidad de Granada email: rubenlopezpico@ugr.es

RESUMEN: En el presente texto se reproduce la Crónica del Congreso Internacional e Interdisciplinar italo-español "L'Unione europea, il Diritto processuale e il Diritto amministrativo locale: la difesa dei diritti e degli interessi dei cittadini”, que se llevó a cabo en Bolonia durante los días 25 y 26 de mayo de 2017. Su objetivo fundamental fue estudiar el impacto del Derecho europeo en el Ordenamiento procesal de los distintos estados miembros, particularmente en el caso de España e Italia, así como en el Derecho administrativo local de los mismos.

PALABRAS CLAVE: Crónica de Congreso, Derecho comunitario, Derecho procesal de los Estados Miembros, Derecho administrativo local.

ABSTRACT: This text reproduces the Conference review of the Spanish-Italian International and Interdisciplinary Congress ' $L$ 'Unione europea, il Diritto processuale e il Diritto amministrativo locale: la difesa dei diritti e degli interessi dei cittadini”, which was held in Bologna from 25 to 26 May 2017, whose main objective was to study the impact of European law on the procedural order of the Member States, in particular Spain and Italy, as well as on local administrative law.

KEY WORDS: Conference review, Community law, National procedural law of the Member States, Local administrative law. 


\section{PLANTEAMIENTO.}

Los días 25 y 26 de mayo de 2017 tuvo lugar en la ciudad de Bolonia (Italia) el Congreso Internacional e Interdisciplinar italo-español " $L$ 'unione Europea, il Diritto processuale e il Diritto amministrativo locale: la difesa dei diritti e degli interessi dei cittadini”. El evento se organizó por el Real Colegio de España en Bolonia, en colaboración con el Dipartimento di Scienze Giuridiche de la Universidad de Bolonia y el Consiglio Dell’Ordine Degli Avvocati de Bolonia, integrándose como actividad formativa acreditada en los distintos programas de doctorado de la Universidad de Bolonia y en especial del programa de doctorado en Scienze Giuridiche, así como en el programa formativo profesional del Consiglio Dell’ordine Degli Avvocati de Bolonia.

Fue dirigido por los profesores Paolo Biavati, Catedrático de Derecho Procesal Civil de la Universidad de Bolonia; Elena Zucconi Galli Fonseca, Catedrática de Derecho Procesal Civil de la Universidad de Bolonia; y Lea Querzola, Profesora Titular de Derecho Procesal Civil de la Universidad de Bolonia. En su comité científico participaron igualmente los profesores Giuseppe Piperata, Catedrático de Derecho Administrativo de la Universidad IUAV de Venecia; Eloísa Carbonell Porras, Catedrática de Derecho Administrativo de la Universidad de Jaén; Rafael Cabrera Mercado, Profesor Titular de Derecho Procesal de la Universidad de Jaén y Of Counsel del Bufete de Abogados "Martínez-Echevarría"; y Claudia Tubertini, Profesora Titular de Derecho Administrativo de la Universidad de Bolonia. Los secretarios académicos y coordinadores del evento fueron los doctorandos Pedro Manuel Quesada López y Rubén López Picó.

En su financiación participaron, además de la propia institución del Real Colegio de España, los Proyectos de Investigación $\mathrm{I}+\mathrm{D}+\mathrm{i}$ del Plan Nacional "Las entidades locales, sus relaciones y competencias. Realidad, efectos y consecuencias de la racionalización y sostenibilidad financiera en clave nacional y europea" (DER201674843-C3-1-R) y del Plan de Apoyo a la Investigación, Desarrollo Tecnológico e Innovación de la Universidad de Jaén "La mediación como alternativa a la judicialización de asuntos mercantiles y administrativos. Aspectos sustantivos y procesales" (UJA2014/06/04); así como el Bufete de Abogados "Martínez-Echevarría". 
$\mathrm{El}$ evento académico se orientó principalmente a expertos y estudiosos del Derecho procesal, doctorandos, abogados y demás profesionales del Derecho, y se desarrolló en formato bilingüe italiano y español, contando con una elevada participación de ambas nacionalidades en todos los sectores profesionales.

Durante su duración, el Congreso acogió la participación de prestigiosos profesores de distintas universidades, tanto de España como de Italia. Su desarrollo permitió abordar cuestiones de gran actualidad jurídica en el ámbito civil, procesal, laboral, mercantil, financiero y administrativo, desde un punto de vista nacional y comunitario, así como comparado entre los dos países España e Italia.

El Congreso se estructuró en tres grandes sesiones: "L'impatto del diritto dell'Unione Europea sul Diritto processuale nazionale", "I diritti dei cittadini di fronte alle misure nazionali ed europee di razionalizzazione e sostenibilità finanziaria degli enti local", y "La tutela dei consumatori in Italia, Spagna e Unione europea", además de una "Tavola rotonda di interventi", en la que se defendieron las comunicaciones previamente admitidas por el consejo científico del evento académico.

Las sesiones quedaron divididas en dos jornadas, la primera, el día 25 de Mayo, con una única sesión de tarde, además de la mesa de intervenciones; y la segunda, el día 26 de Mayo, con dos sesiones de mañana separadas por una pausa-café.

\section{JORNADA DEL DÍA 25 DE MAYO DE 2017.}

El desarrollo de esta primera sesión del Congreso tuvo lugar de forma íntegra en la "Sala delle Armi" de la Scuola di Giurisprudenza de la Universidad de Bolonia, continuadora de la facultad de derecho más antigua del mundo.

La inauguración del Congreso corrió a cargo del Adjunto al Delegado Especial de la Junta del Patronato en el Real Colegio de España en Bolonia, el Prof. D. Juan José Gutiérrez Alonso, y de los directores del evento académico Paolo Biavati, Elena Zucconi Galli Fonseca y Lea Querzola. En ella quedo plasmada la importancia que suponía la organización de un evento académico de tales magnitudes, al constituir éste último un punto importante de encuentro e intercambio académico.

Finalizada la inauguración del Congreso, comenzó la primera de las sesiones "L'impatto del diritto dell'Unione Europea sul Diritto processuale nazionale"- con una breve intervención, para dar la bienvenida a los profesores españoles, del Prof. Paolo Biavati, que fue el encargado de presidirla y moderarla. Acto seguido, comenzó la 
intervención del Prof. Michele Angelo Lupoi, Profesor Titular de Derecho Procesal Civil de la Universidad de Bolonia, titulada "I will follow, ovvero come il diritto processuale interno segue gli sviluppi della normativa europea”. A través de ella, el Prof. Lupoi, se centró en mostrar como el Derecho Procesal interno de los distintos estados miembros ha quedado influenciado por el Derecho de la Unión Europea. Influencia que ha tenido lugar a través del art.7 del Convenio Europeo de Derechos Humanos, y que se ha vertido de forma especial sobre la jurisprudencia y la legislación. Sobre la jurisprudencia, al dictar el Tribunal de Justicia de la Unión Europea (TJUE) y el Tribunal Europeo de Derechos Humanos (TEDH) dan lugar a sentencias de carácter vinculante para el poder judicial de los distintos estados miembros. Y respecto a la legislación, mediante el reconocimiento, en forma de sustitución de las normas de derecho interno de los distintos estados europeos por las de la Unión Europea (UE), como resultado de la incompatibilidad entre las mismas, para alcanzar un cierto grado de armonización sobre determinados aspectos jurídicos en todos los países miembros de la UE.

Tras esta intervención, le correspondió la palabra a la Prof. ${ }^{a}$ Lea Querzola, quién a través de su intervención titulada "L'impatto del diritto europeo in materia cautelare", abordó las normas y los diferentes aspectos del derecho italiano en materia de medias cautelares, y las incidencias que éste último había experimentado a raíz de la influencia ejercida por el derecho europeo sobre el mismo.

En tercer lugar, intervino el Prof. Juan Herrero Perezagua, Catedrático de Derecho Procesal de la Universidad de Zaragoza, para abordar con su ponencia "Le modifiche alla Legge sulla Procedura Civile spagnola in applicazione del Diritto europeo". El Prof. Perezagua puso de manifiesto como en los últimos diecisiete años 2000/2017-, la Ley de Enjuiciamiento Civil ha sido objeto de, aproximadamente, unas cuarenta reformas debido a la influencia del derecho europeo, modificando las normas jurídicas y la jurisprudencia. Entre todas esas reformas, hizo referencia a cinco que, a su juicio, presentaban un mayor interés: la primera respecto a la acción de cesación; la segunda respecto a la protección de los derechos de protección industrial e intelectual; la tercera respecto a la aplicación de las normas comunitarias de competencia y cooperación entre autoridades; la cuarta respecto a la acción de derecho de daños, que permite el acceso a aquellas pruebas que no se encuentran al alcance de la ciudadanía, lo que se traduce en un acercamiento a los procesos anglosajones de la institución del discovery; y el quinto respecto a los cambios experimentados en el proceso de ejecución 
hipotecaria y en el proceso monitorio mediante la incorporación de disposiciones finales y adicionales a la Ley de Enjuiciamiento Civil española. Reformas que en todo caso y como conclusión esencial del ponente han tenido lugar con el fin de adaptar el derecho interno español al de la UE, para acabar así con las incompatibilidades existentes entre ambos.

Por último, esta primera sesión se clausuró con la participación del Prof. Jordi Nieva Fenoll, Catedrático de Derecho Procesal de la Universidad de Barcelona, quién se decantó por la cuestión relativa a "L'attività d'ufficio del giudice civile nazionale nell'Unione europea". A través de ella, abordó el concepto o la idea original que existía en el Derecho nacional español del principio dispositivo y su reciente matización motivada por la jurisprudencia europea. El ponente señaló como ésta última, a través de la aplicación de la Directiva 93/13 ha influenciado, y posteriormente modificado dicho principio, que adquiere un especial interés en el ámbito del derecho de consumo al permitir que el juez pueda actuar e intervenir de oficio en el proceso civil para evitar situaciones de abuso sobre los derechos de los consumidores.

Tras un breve descanso, para poner fin a la primera jornada del Congreso, tuvo lugar el módulo de comunicantes, desarrollado a través de una "tavola rotonda di interventi", donde los doctorandos Rubén López Picó y Pedro Manuel Quesada López, de la Universidad de Granada y Universidad de Jaén, respectivamente, además de intervenir con las ponencias "Il procedimento europeo d'ingiunzione di pagamento" y "L'incidenza dei principi del diritto europeo nel procedimento spagnolo di esecuzione ipotecaria", fueron los encargados de moderarla y presidirla.

La mesa destacó, por el elevado número de comunicaciones, así como por el altísimo nivel jurídico alcanzado, girando dichas intervenciones en torno a temáticas tan variadas como las siguientes: "La interpretación del derecho europeo por el tribunal de justicia de la unión europea y los cambios legislativos y jurisprudenciales en España en materia de protección de los consumidores y usuarios", a cargo de la Prof. a Carmen Vallejo Peña -Universidad de Jaén-, "La tutela procesal de los datos personales en el nuevo reglamento 2016/679 UE”, por el Prof. Enrique Pérez-Luño Robledo Universidad de Sevilla-, "Diritto a un ricorso effettivo: studio del regime giuridico del diritto a un equo processo nell'Unione Europea. Attenzione particolare al giudice imparziale, presunzione di innocenza, principi della legalità e della proporzionalità e il loro rapporto con il diritto del lavoro", realizada por el Prof. Pompeyo G. Ortega Lozano -Universidad de Granada-,"Los recursos masivos en el derecho procesal 
europeo", de la mano de la Prof. a María Luisa Domínguez Barragán -Universidad de Sevilla-"'La tutela del consumidor: protección de consumidores y usuarios en materia de publicidad engañosa en la Unión Europea. Legitimación activa en el ámbito del delito publicitario", por la Prof. a Belén Macías Espejo -Universidad de Jaén-,"Il principio rogatorio nelle procedure concorsuali: gli ordinamenti spagnolo e italiano a confronto", a cargo del Dr. Gabriel A. García Escobar -Universidad de Granada-,"El diálogo entre el TJUE y los tribunales nacionales como fenómeno de derecho global”, del doctorando Héctor Iglesias Sevillano -Universidad Autónoma de Madrid-, "La agregación de la demanda de las entidades locales como instrumento de sostenibilidad económica de la contratación pública", de manos del doctorando Alfonso Sánchez García -Universitá di Bologna-, y "La prova del documento elettronico nella nuova normativa europea in materia di identificazione elettronica e servizi fiduciari”, del doctorando Juan Francisco Rodríguez Ayuso -Universitá di Bologna-.

\section{JORNADA DEL DÍA 26 DE MAYO DE 2017.}

Por lo que respecta a la segunda jornada del Congreso, la misma tuvo lugar en dos sesiones en la Casa de Cervantes del Real Colegio de España en Bolonia.

La primera de las sesiones giró en torno a "I diritti dei cittadini di fronte alle misure nazionali ed europee di razionalizzazione e sostenibilità finanziaria degli enti local", y estuvo moderada por la Prof. ${ }^{a}$ Eloisa Carbonell Porras, quién destacó la enorme vinculación del argumento de esta sesión con múltiples aspectos de la sociedad y del derecho. Posteriormente tomó la palabra la Prof. ${ }^{\text {a }}$ Marina Caporale, Profesora Titular de Derecho Administrativo en la Universidad de Bolonia. Se centró en reseñar los aspectos más importantes que habían sido modificados en Italia, a raíz de las distintas reformas producidas como resultado de la crisis económica, a fin de cumplir con los objetivos económicos, de déficit y de sostenibilidad económica impuestos desde las instituciones europeas. Reformas y cambios que han originado un importante debate, al afectar fundamentalmente, la mayoría de ellas, a las administraciones públicas de la provincia y la ciudad metropolitana. Destacó de forma especial, entre ese conjunto de reformas, la del sistema de elección de los cargos electorales de estos territorios, que queda regulado a través de un nuevo sistema electoral de segundo nivel. 
Tras esta primera intervención, llegó el turno de la Prof. a Carmen Cámara Barroso, Profesora Contratada Doctora de Derecho Tributario de UDIMA de Madrid, que analizó un tema de reciente actualidad jurídica y enorme importancia económica como son las plusvalías municipales. Y es que el Tribunal Constitucional con su reciente sentencia de 11 de mayo de 2017 ha determinado que el pago de este impuesto en aquellos casos en los que la transmisión que se realice tenga lugar con pérdidas es inconstitucional de acuerdo al contenido de los artículos 24 y 31 de la Constitución Española, aspecto profundizado y analizado por la ponente.

Recogió el testigo el Prof. Giuseppe Piperata para abordar la pérdida de autonomía de las administraciones italianas, especialmente de la administración local, como resultado de las distintas reformas soportadas por las mismas en los últimos años a raíz de la crisis económica. Para ello, comenzó abordando el papel de la administración local en el siglo XIX, destacando su papel protagonista ante la incapacidad de la administración estatal y regional para dar respuesta a las necesidades de la ciudadanía, lo que le fue merecedor del reconocimiento constitucional de su autonomía local. Situación radicalmente opuesta a la actual en el sistema transalpino, dónde como resultado de la fuerte crisis económica y de todas las reformas que ésta última ha llevado aparejada de forma implícita, la administración local ha visto afectada su autonomía al pasar de un sistema federal -Legge 26 marzo 2010- a un sistema centralizado no solo por la crisis económica sino también por el papel de la empresa privada -interesada en prestar servicios de carácter público- y la potenciación de las instituciones europeas. Lo que se ha traducido finalmente en la reducción de la esfera pública, la imposición a nivel europeo, el cambio del rol de la administración pública, y el sometimiento de ésta última al poder central, surgiendo un importante debate en torno a si todas estas reformas suponen finalmente un beneficio o un perjuicio para los intereses de los ciudadanos de la Unión Europea.

El cierre de la segunda sesión corrió a cargo de la Prof. ${ }^{a}$ Claudia Tubertini, encargada de tratar la cuestión de los derechos sociales y la jurisprudencia italiana sobre éstos. Según la Prof. ${ }^{\text {a }}$ Tubertini las diferentes reformas llevadas a cabo -de manera especial a través de la Legge 224/2015- en el ámbito de la administración pública para alcanzar los objetivos de racionalidad y sostenibilidad económica impuestos desde las instituciones europeas a los distintos estados miembros para hacer frente a la crisis económica, han supuesto la reducción de la capacidad económica, y por tanto de la capacidad de actuación de la administración local a la hora de poder continuar prestando 
sus servicios sociales, lo que se traduce directamente en una merma de los derechos y servicios sociales reconocidos a la ciudadanía europea.

Tras una pausa-café, comenzó la segunda sesión, en la que se trató "La tutela dei consumatori in Italia, Spagna e Unione europea", moderada por la Prof. ${ }^{a}$ Elena Zucconi Galli Fonseca. Tras una breve introducción de la Prof. ${ }^{\text {a }}$ Zucconi, tomó la palabra el Prof. Andrea Giussani, Catedrático de Derecho Procesal Civil en la Universidad Carlo Bo de Urbino, para disertar entorno a "Le azioni collettive e di classe", centrándose esencialmente en la utilización de la acción colectiva como un mecanismo, herramienta o instrumento orientado a la consecución de la tutela resarcitoria de los derechos e intereses de los ciudadanos europeos.

A continuación se dio paso al Prof. Eugenio Dalmotto, Profesor Titular de Derecho Procesal Civil de la Universidad de Turín, quien a través de su ponencia sobre “L’arbitrato dei consumatori” manifestó su posición en relación a la utilización del arbitraje como un mecanismo orientado a la protección de los derechos e intereses de los ciudadanos europeos, especialmente en materia de consumo. El Prof. Dalmotto señaló como la utilización del arbitraje en materia de consumo presenta, a día de hoy, dos grandes inconvenientes: la fuente del arbitraje y la eficacia del laudo arbitral. En relación al primero de ellos, la fuente del arbitraje, destacó el carácter voluntario del arbitraje sobre todo en las fases previas al proceso judicial, y la ineficacia de la cláusula de sometimiento arbitral en materia de contratos de consumo al considerarse abusiva, salvo que se demuestre de forma previa su carácter negociado entre ambas partes enfrentadas. Por otro lado, en cuanto a la eficacia del laudo arbitral, explicó como en la norma italiana en materia de consumo el laudo arbitral no es vinculante cuando enfrenta al empresario y al consumidor, al entender que el consumidor no puede quedar impedido de acudir a la vía judicial para que ésta última tutele su derecho en caso de que el laudo previamente emitido no le sea beneficioso. De modo, que el laudo arbitral solo será vinculante para el consumidor cuando éste así lo estipule. Por ello la conclusión principal fue que la norma italiana apuesta de forma clara por una postura a favor de la defensa y protección del consumidor en la regulación de las relaciones arbitrales.

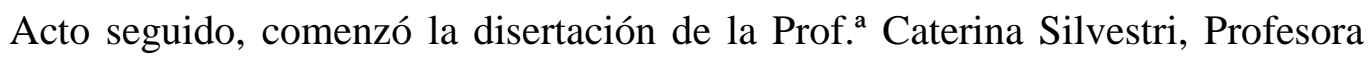
Profesora Asociada de Derecho Procesal Civil de la Universidad de Florencia, con el título "La nozione di consumatore nel sistema di Bruxelles II". En su intervención, la Prof. a Silvestri abordó la cuestión del reconocimiento y la ejecución de las decisiones 
judiciales en materia de consumo a fin de garantizar la protección de los derechos e intereses de los ciudadanos europeos.

El siguiente ponente fue el Prof. Ignacio Gallego Domínguez, Catedrático de Derecho Civil de la Universidad de Córdoba y Socio Director del Departamento de Derecho Civil del Bufete de Abogados "Martínez-Echevarría". A través de su ponencia "Le clausole abusive. Influenza della legislazione europea in materia di tutela dei consumatori e la giurisprudenza della Corte di giustizia dell'Unione europea in Spagna", trató un tema de enorme actualidad e importancia jurídica en España: las cláusulas suelo. Las sentencias del TJUE en contra de las normas de derecho interno español en materia de cláusulas suelo -al considerar éstas últimas como abusivas y por tanto no vinculantes para el consumidor-, resultado de la previa formulación de una cuestión prejudicial por un juez español, puso de manifiesto la prueba más evidente de la supremacía del Derecho Europeo sobre las normas nacionales en materia de consumo, y más concretamente en materia de cláusula suelo. Sin embargo, ésta no es la única sentencia del TJUE que para conseguir la real y efectiva protección de los derechos e intereses de los consumidores europeos va en contra de las normas de derecho interno español. Éste también es el caso de las sentencias de 26 de octubre de 2006, 3 de junio de 2013, 14 de marzo de 2013, 5 de diciembre de 2013, 16 de enero de 2014, y 21 de diciembre de 2016, resultado de la previa formulación de diversas cuestiones prejudiciales por parte de los jueces españoles.

En quinto lugar, se trató "La tutela giurisdizionale dei diritti dei consumatori e la possibile applicazione della mediazione in questo ambito", de la mano del Prof. Rafael Cabrera Mercado. En esta ponencia se abordó en profundidad la utilización de la mediación como un instrumento orientado a la tutela judicial de los derechos e intereses de los consumidores europeos, haciéndose especial referencia al Reglamento 524/2013, regulador de la plataforma europea extrajudicial de resolución de litigios en línea en materia de consumo. Una plataforma que a pesar de disponer de un procedimiento de resolución de litigios en materia de consumo ágil, rápido y sencillo, y dotado de todas las garantías y principios básicos, apenas ha sido utilizado en un primer año de vigencia, pues durante el desarrollo de éste solo se han resuelto aproximadamente unos cuarenta mil litigios.

La clausura de la sesión, y por tanto del Congreso, tuvo lugar con la intervención "L'indefinitezza del sistema di azioni collettive in Spagna ed in Europa" del Prof. Javier López Sánchez, Profesor Titular de Derecho Procesal de la Universidad de Zaragoza, 
que giró en torno a la acción colectiva en el caso español, y en el marco de la Unión Europea. Comenzó explicando como la figura de la acción colectiva surgió en Estados Unidos para luego ser recepcionada en el ámbito europeo. El desarrollo de las primeras acciones colectivas en este último ámbito, se ha caracterizado por la presencia de un cierto grado de temor a la hora de llevar a cabo su regulación, como resultado de la identificación de las acciones colectivas por parte de las instituciones de la Unión Europea como un modelo de negocio, una fuente de intereses económicos, al dar lugar a los denominados fondos "Robin Hood": sujetos con gran capacidad económica que conceden importantes cantidades de dinero a grandes despachos de abogados para que pleiteen y defiendan los derechos e intereses de los ciudadanos que no disponen de los fondos económicos necesarios con lo que poder articular la reclamación de sus derechos e intereses ante los tribunales de justicia. A cambio, o con la condición, de que en caso de obtener la "razón" por parte de los tribunales de justicia, se les conceda una parte de los posibles beneficios que se pudiesen obtener. Situación, que de acuerdo al criterio del TJUE sería contraria al principio de efectividad, al tener el ciudadano europeo, inmerso en este tipo de procesos colectivos, que esperar a que los tribunales de justicia resuelvan para poder ver protegidos sus derechos e intereses, como ha puesto de manifiesto la reciente Sentencia del Tribunal de Justicia de la Unión de 14 de abril de 2016. 